\title{
Deposição por Plasma com Arco Transferido
}

\author{
(Hardfacing by Plasma Transfer Arc Process)
}

\author{
Víctor Vergara Díaz', Jair Carlos Dutra², Ana Sofia Clímaco Monteiro D’Oliveira ${ }^{3}$ \\ ${ }^{1}$ Universidad de Antofagasta, Departamento Engenharia Mecânica, Antofagasta, Chile, vvergara@uantof.cl \\ ${ }^{2}$ Universidade Federal de Santa Catarina, Departamento de Engenharia Mecânica, Florianópolis, Santa Catarina, Brasil, jdutra@ \\ labsolda.ufsc.br \\ ${ }^{3}$ Universidade Federal do Paraná, Departamento de Engenharia Mecânica, Curitiba, Paraná, Brasil, sofmat@ufpr.br
}

\begin{abstract}
Resumo
Em virtude do Processo de Soldagem Plasma com Alimentação de Pó ter similaridades com o Processo de Soldagem Plasma com Alimentação de Arame, foi realizado um estudo comparativo entre ambos os processos utilizando-se a liga a base de cobalto comercialmente conhecida como Stellite 6, como material de adição na forma de pó e arame. A pesquisa foi realizada com a expectativa de ser aplicada nas operações de revestimentos de superfícies, em especial em pás de turbinas hidráulicas desgastadas por cavitação. A seleção do material de adição a ser empregado depende da natureza do mecanismo de desgaste encontrado. No Labsolda, a liga Stellite 6 vem sendo uma das mais utilizadas, por apresentar uma excelente resistência ao desgaste erosivo por cavitação. Foi avaliada a influência da vazão de gás de plasma a partir dos valores de diluição, dimensões do cordão, dureza e microestrutura. O Processo de Soldagem Plasma com Alimentação de Pó foi o que produziu o melhor acabamento superficial, menor diluição, melhor molhamento e maior largura. Com isto abre-se uma nova perspectiva para revestimentos metálicos e neste contexto se insere a recuperação por soldagem de partes erodidas de turbinas hidráulicas.
\end{abstract}

Palavras-chave: Soldagem Plasma; Arco Transferido; Diluição; Stellite 6

Abstract: The Plasma powder transferred arc welding process, which uses feed stock in the powder form, has similarities with Plasma wire transferred arc welding. This work describes a comparative study of the two processes using a Cobalt-based alloy commercially known as Stellite 6. This Co-based alloy is recognized for its superior cavitation erosion resistance. The aim of this work is to investigate the potential of PTA coatings for the protection and refurbishiment hydraulic turbine blades. Coatings were evaluated for the influence of Plasma gas flow rate on coating dilution, geometry, hardness and microstructure. Coatings processed with the atomized Stellite 6 powder feestock showed a superior surface quality, lower dilution, better wettability and wider tracks. This study provided a novel information about the refurbishing worn hydraulic turbines blades, leading to a longer service life.

Key-words: Welding plasma; Transferred Arc; Dilution; Stellite 6

\section{Introdução}

De acordo com a literatura, o processo de soldagem Plasma de Arco Transferido que utiliza metal de adição na forma de arame é chamado de PAW (Plasma Arc Welding) e quando o processo de soldagem utiliza pó como material de adição, o processo é usualmente chamado de PTA (Plasma Transferred Arc) [1].

O Processo PTA pode ser considerado uma derivação do processo PAW. Da Figura 1, pode-se observar as similitudes entre os dois processos. Ambos os processos de soldadura utilizam um eletrodo de tungstênio não consumível que fica no

(Recebido em 15/11/2008; Texto Final em 08/12/2009). interior da tocha, um bico constritor refrigerado a água, gás de proteção para a poça fundida e o gás de plasma. A diferença entre ambos os processos de soldagem está na natureza do material de aporte, pó em vez de arame, o qual requer de um gás para o seu transporte até a região do arco. A Figura 1 mostra um esquema de ambos os processos, com suas diferenças e similitudes.

Os equipamentos necessários para a execução de depósitos através do processo plasma PTA são muito similares aos empregados no processo plasma PAW. Quando é utilizado o processo PAW é necessário um equipamento capaz de tracionar arames bobinados de diversas bitolas e de diferentes materiais, com velocidades constantes ou pulsadas. Já no Processo de Soldagem Plasma PTA, o consumível usado é na forma de pó, e para poder ser transportado até o arco voltaico para produzir o revestimento é necessário de um equipamento específico denominado de alimentador de pó.

Do ponto de vista de sua aplicação para revestimento, o 
processo PTA se apresenta adequado porque produz valores de diluição da ordem de 6 a $10 \%$ [2], muito inferior ao que se obtém com outros processos de soldagem a arco da ordem de 20 a $25 \%$. A baixa distorção, pequena zona afetada pelo calor e microestrutura refinada também são destaques desta técnica $[3,4]$.

No processo PTA e PAW como gás de plasma é utilizado um gás inerte, que é forçado a passar através do orifício do bico constritor, onde o eletrodo é fixado concentricamente. O gás de proteção, passa por um bocal externo, concêntrico ao bico constritor, atuando, efetivamente na proteção da solda contra a contaminação pelo ar atmosférico (pode ser ativo ou inerte). Já para o processo PTA, como meio de transporte do pó é utilizado um gás chamado de gás de arraste ou de transporte utilizado para carrear o material consumível, mediante mangueiras flexíveis até o bico constritor, permitindo a entrada em forma convergente no arco plasma. $\mathrm{O}$ gás geralmente utilizado é argônio.

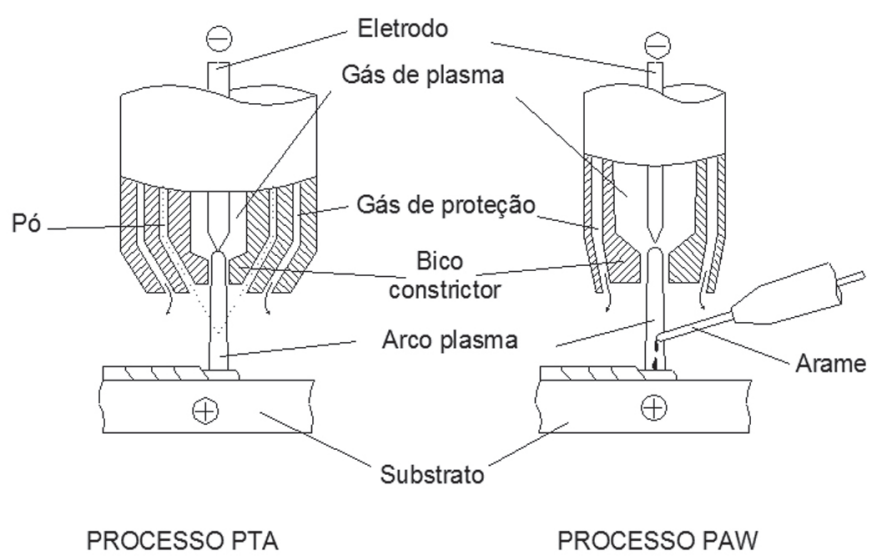

Figura 1. Comparação entre os processos Plasma de Arco Transferido PTA e PAW.

Devido ao fato do eletrodo de tungstênio ficar no interior do bico constritor da tocha de soldagem, torna-se difícil a abertura do arco por contato, sendo então indispensável a utilização de um equipamento, denominado de módulo plasma, para estabelecer a abertura do arco. Um ignitor eletrônico fornece picos de tensão entre o eletrodo de tungstênio e o bocal constritor, gerando um pequeno faiscamento nesta região. Assim, com a passagem do gás de plasma surge um arco elétrico de baixa intensidade entre o eletrodo de tungstênio e o bocal constritor, chamado de arco piloto (arco não-transferido). Por sua vez, o arco piloto forma um caminho de baixa resistência elétrica entre o eletrodo de tungstênio e a peça a ser soldada facilitando o estabelecimento do arco principal quando a fonte de potência for acionada.

Na prática, os parâmetros que controlam a qualidade da solda são taxa do material de adição, vazão de gás (gás de proteção, gás de plasma, gás de transporte), corrente de soldagem, distância bico-peça e velocidade de soldagem.

A configuração básica do bico constritor é mostrada na Figura 2, onde estão indicados os parâmetros empregados no processo. A distância da face externa do bico constritor até o substrato é a chamada de distância bico-peça (DBP).

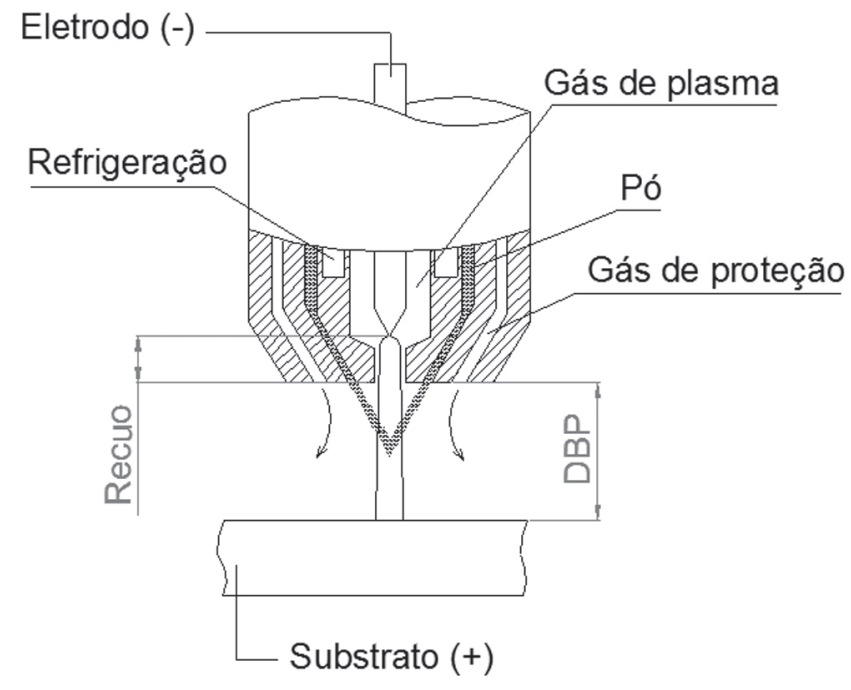

Figura 2. Distância Bico-Peça (DBP) e Recuo do Eletrodo (Rc) [6].

O recuo (Rc) do eletrodo é medido desde a ponta do eletrodo até a face externa do bico constritor. As alterações das características do arco são influenciadas por este fator, o qual define o grau de constrição e a rigidez do jato plasma [5].

Oliveira [5] estudou a influência do recuo do eletrodo no processo plasma de arco transferido alimentado com arame, objetivando identificar uma possível influência do grau de constrição do arco sobre a tensão do arco. Os resultados mostraram em média uma variação da tensão em função do recuo do eletrodo de $2,4 \mathrm{~V} / \mathrm{mm}$.

Normalmente, os valores máximo e mínimo para ajuste de recuo do eletrodo variam conforme o modelo da tocha de soldagem. A titulo de exemplo, a tocha de soldagem PWM-300, fabricada pela Themal Dynamics Corporation, possui uma faixa de ajuste para o recuo do eletrodo que varia de 0,8 a $2,4 \mathrm{~mm}$.

$\grave{A}$ medida que se reduz o recuo do eletrodo, a largura do cordão aumenta e são obtidos cordões com menor profundidade de penetração. Esta variação nas características geométricas do cordão de solda se deve à redução do efeito de constrição, produzindo uma maior área de incidência do arco sobre o substrato.

O bico constritor, de cobre, onde o eletrodo fica confinado, apresenta um orifício central pelo qual o arco e todo o volume de gás de plasma passam. O diâmetro do orifício do bico constritor tem uma grande influência sobre a qualidade do revestimento devido a que está relacionado diretamente com a largura e penetração do cordão produzido. Uma vazão de gás de plasma deficiente afeta a vida do bico constritor produzindo seu desgaste. A corrente de soldagem fica mais reduzida a medida em que são utilizados menores diâmetros de orifícios constritores, devido ao aumento de temperatura do arco de soldagem.

O grau de influência da distância bico-peça sobre o revestimento depende muito do recuo de eletrodo em relação ao bico constritor e do diâmetro do orifício constritor. Quanto maior é o recuo adotado para o eletrodo e menor o diâmetro do orifício constritor, maior é o efeito de constrição do arco, 
tornando-se mais concentrado.

$\mathrm{Na}$ técnica "melt-in" se utilizam pequenos valores de recuo do eletrodo, sendo o arco submetido a um pequeno grau de colimação, assumindo uma forma cônica. Nesta situação, a variação da distância bico-peça, mesmo dentro dos limites normais, resulta na alteração das características do cordão de solda, do mesmo modo que ocorre no processo TIG. Desta maneira, quanto maior é a distância bico-peça, menor a penetração e maior largura do cordão devido ao aumento da área de incidência do arco sobre o substrato.

Hallen et al. [7] relatam que para poder obter um bom rendimento de deposição, a distância bico-peça não deve ser maior do que 10 a $15 \mathrm{~mm}$. Para valores acima desta faixa, a eficácia da proteção gasosa é reduzida significativamente.

Os autores deste artigo apresentam também resultados em relação à distância bico-peça para dois níveis: 15 e $20 \mathrm{~mm}$. O estudo mostra que a medida que aumenta a distância bico-peça o grau de diluição diminui.

O presente trabalho tem como objetivo geral o estudo do processo de soldagem PAW e PTA com a expectativa de ser aplicado nas operações de revestimentos de superfícies, em especial em pás de turbinas hidráulicas desgastadas por cavitação. Este trabalho foi motivado por ter-se observado escassa informação em relação aos benefícios que apresenta o processo de soldagem plasma com pó frente ao processo de soldagem plasma com arame na aplicação em revestimentos. São avaliadas as características geométricas dos cordões, grau de diluição, dureza e microestrutura.

\section{Materiais e Métodos}

\subsection{Bancada de ensaios}

Inicialmente foi montada uma bancada a partir de equipamentos já desenvolvidos no próprio LABSOLDA [5, 6] que permitiu fazer ensaios com o processo a plasma por arco transferido alimentado com arame. Logo, na mesma bancada, foi montado o processo de soldagem a plasma de arco transferido alimentado com pó. A fonte de soldagem é um equipamento que, através de uma interface, foi conectada a um microcomputador. Por intermédio de um software bastante versátil, quase todas as variáveis do processo podem ser comandadas.

Dos três circuitos de gás, o que foi dado maior atenção foi o gás de plasma pela sua grande relevância na qualidade dos depósitos. Foi usado um controlador de vazão mássica, na qual, o controle é realizado eletronicamente e o sinal de regulagem é uma tensão de referência. Os outros circuitos de vazão de gás são simplesmente monitorados por fluxômetros eletrônicos, porém volumétricos.

Uma das partes fundamentais do equipamento é o dispositivo denominado de móduloplasma, oqual viabiliza a soldagem plasma em qualquer versão a partir de fontes de soldagem convencionais para TIG ou eletrodo revestido. Para o deslocamento da tocha de soldagem foi utilizado um dispositivo eletrônico denominado de TARTÍLOPE. O componente do sistema, que foi integralmente projetado para este desenvolvimento específico, foi o dispositivo de alimentação de pó, que funciona com a combinação de um parafuso sem fim e de um fluxo de gás como mecanismos para carrear o pó. A tocha de soldagem foi um desenvolvimento a partir de uma tocha plasma para keyhole. A grande vantagem está no aspecto multiprocesso da mesma, que permite, ora trabalhar com o plasma com pó e ora que com plasma convencional. Além disso, o projeto de adaptação permite a utilização de bicos constritores de plasma com diferentes ângulos de convergência para a alimentação do pó. Inicialmente foi realizado um análises nas tochas a ser utilizadas nesta pesquisa. Observou-se que a tocha PTA, possui um bico com diâmetro constritor de 4,8 mm. Em relação à tocha $\mathrm{PAW}$, o fabricante fornece três bicos com diâmetros constritores de 2,4;2,8; e 3,2 mm, os quais estão desenhados em função da corrente de soldagem a ser utilizada.

Neste caso, a decisão foi de trabalhar com o bico de maior diâmetro constritor disponível da tocha PAW, ou seja, de 3,2 $\mathrm{mm}$.

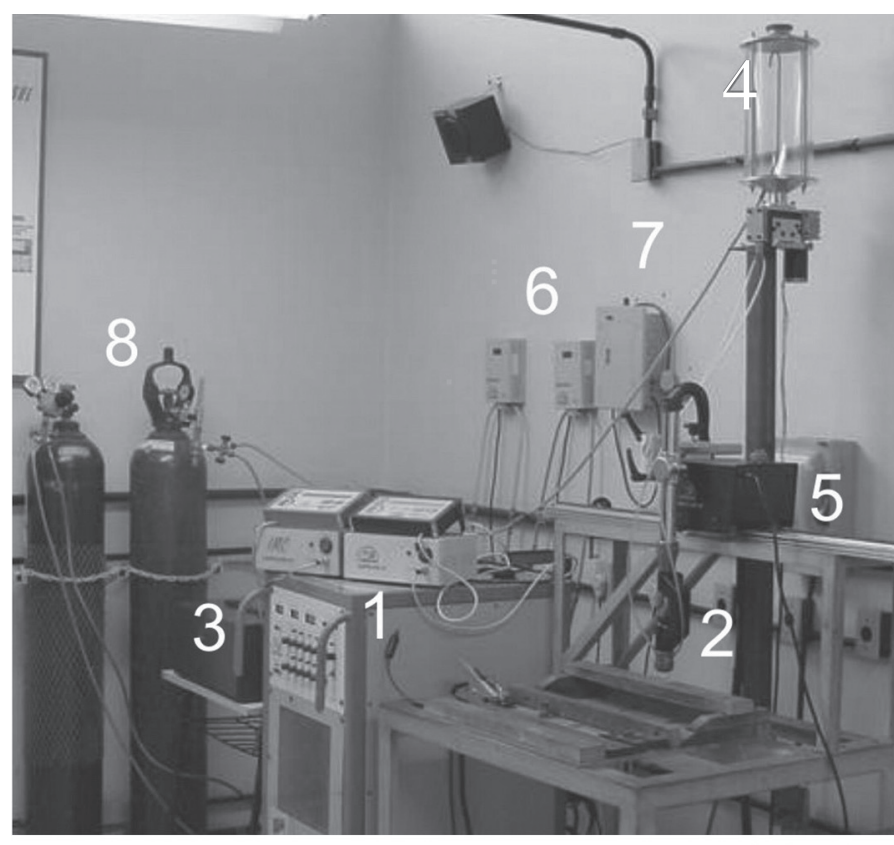

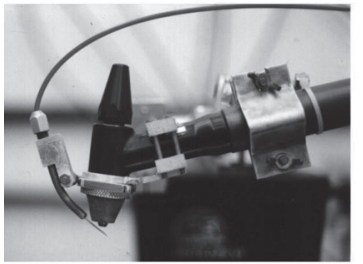

Tocha PAW adaptada

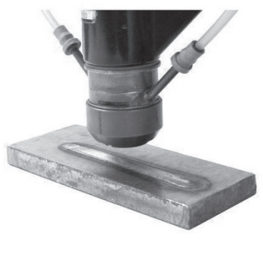

Tocha PTA adaptada

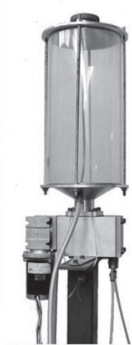

Alimentador de Pó
1-Fonte de soldagem; 2-Tocha Plasma adaptada; 3-Modulo plasma; 4-Alimentador de pó; 5-Sistema de deslocamento da tocha; 6-Medidores digitais de gases; 7-Válvula eletrônica de gás; 8-gases

Figura 3. Montagem da Bancada no laboratório de Soldagem 
A Figura 3 apresenta uma visão geral do equipamento desenvolvido, na parte inferior da figura são mostrados os equipamentos que fazem parte da bancada para o processo de soldagem PAW e PTA.

Neste trabalho foi utilizado argônio com pureza de 99,99 \% para os gases de plasma, proteção e de transporte. Utilizou-se um eletrodo de tungstênio com $2 \%$ de óxido de tório (EWTh-2) com diâmetro de $4,8 \mathrm{~mm}$. O ângulo da ponta do eletrodo foi mantido em $30^{\circ}$ para todos os experimentos.

\subsection{Bico constritor do processo PTA.}

A configuração do bico constritor desenvolvida neste trabalho inclui dois conduítes de passagem de gás de arraste, cujo objetivo é de alimentar com pó o arco plasma, em forma convergente. A Figura 4 mostra o corte transversal do bico constritor. O bico constritor de $60^{\circ}$ permite a entrada de pó diretamente na poça fundida, quando é utilizada uma distância bico-peça de $10 \mathrm{~mm}$.

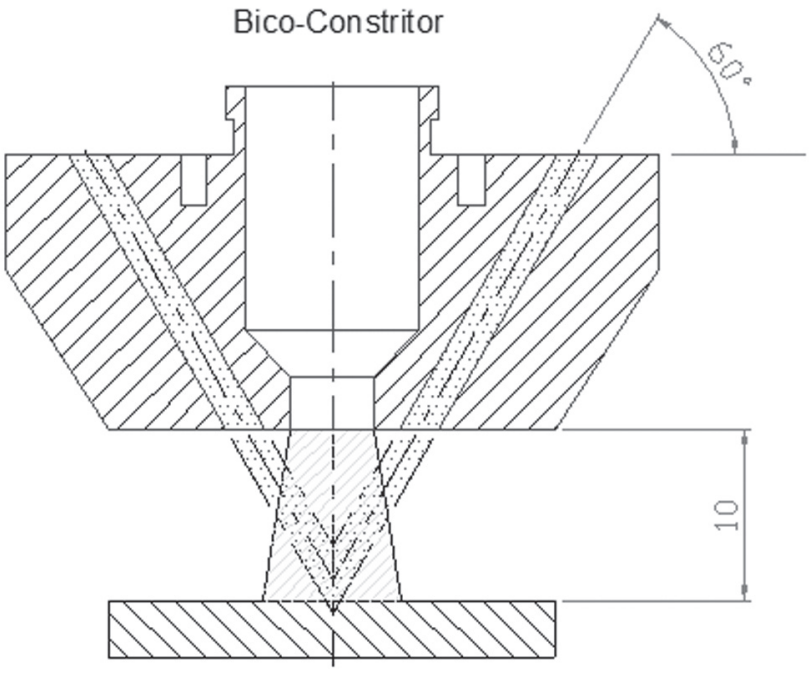

Substrato

Figura 4. Corte transversal do bico constritor mostrando a entrada do fluxo de pó no arco plasma [6]

\subsection{Caracterização}

Depósitos da liga Stellite 6 atomizada, Figura 5, foram processados sobre chapas de aço carbono da classe ABNT 1020 de dimensões $12,5 \times 60 \times 155 \mathrm{~mm}$, utilizando-se corrente continua constante. A Tabela 1 apresenta a composição química do substrato. A análise química dos diferentes materiais de aporte foi realizada através da técnica de espectrometria de emissão ótica e está apresentada nas Tabelas 2 e 3.

Foram depositados cordões unitários com os parâmetros indicados na tabela 4 e amostras foram retiradas para a sua caracterização. Nesta tabela são apresentados os parâmetros operacionais para o processo de soldagem plasma PTA e PAW, na qual, se tem parâmetros que não podem permanecer constantes em ambos os processos, por exemplo: Natureza do material de aporte, no PAW foi arame e no PTA foi pó; velocidade de arame, parâmetro no necessário no processo PTA; gás de transporte, parâmetro no necessário no processo PAW; diâmetro do bico constritor, no PTA o diâmetro foi de $4,8 \mathrm{~mm}$ e no processo PAW o diâmetro foi de $3,2 \mathrm{~mm}$.

Inicialmente os cordões foram submetidos a inspeção visual quanto a presença de defeitos de soldagem, o grau de diluição foi determinado pelo método das áreas em uma macrografia da seção transversal dos depósitos, ataque com nital 6\%. Perfis de microdureza Vickers, com carga de 500 g, permitiram avaliar a uniformidade dos cordões processados, seguindo neste procedimento a norma ABNT6672/81. O levantamento dos perfis de microdureza, média de três medidas, foi feito no centro dos cordões e na região de sobreposição dos mesmos. Para avaliação da microestrutura por microscopia ótica, a preparação da seção transversal seguiu procedimentos padrão sendo a microestrutura revelada após ataque eletrolítico com ácido oxálico.

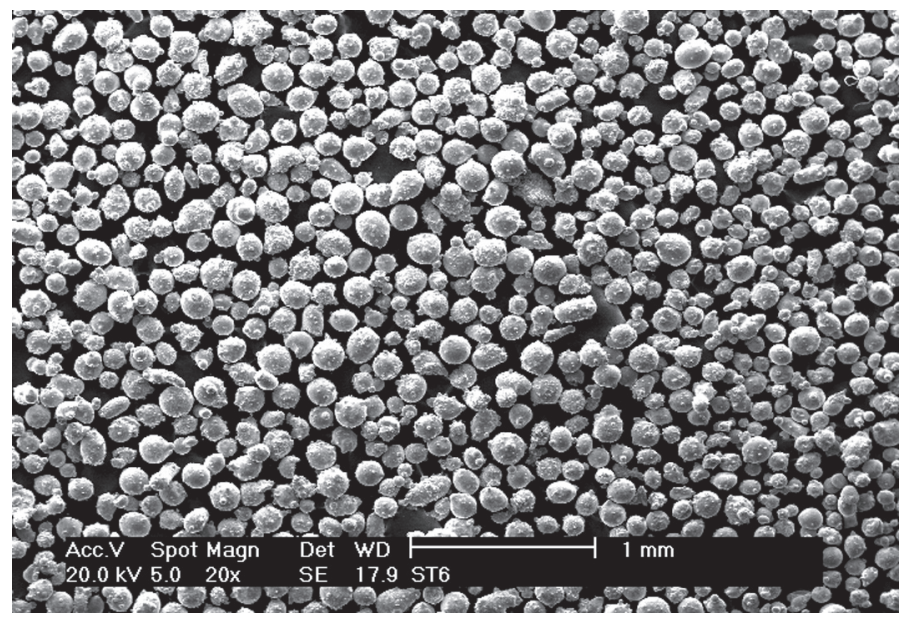

Figura 5. Morfologia do pó depositado pelo processo PTA (Stellite 6).

\section{RESULTADOS E DISCUSSAO}

\subsection{Caracterização geral}

A Figura 6 mostra o aspecto externo dos cordões onde é possível observar diferenças significativas entre eles. O processo PTA foi o que produziu o melhor acabamento superficial, menor diluição, melhor molhamento e maior largura.

As Figuras 7 e 8 mostram a seção transversal dos cordões obtidos pelos dois processos (PAW e PTA) onde é possível observar diferenças significativas no perfil de penetração dos cordões e a Figura 9 apresenta os resultados das condições geométricas dos cordões, para três níveis de vazão de gás de plasma testados neste estudo: 2,2; 2,4 e 3,0 1/min. Quando comparados os depósitos realizados através dos dois processos, pode-se observar que o reforço e a penetração são sempre menores no processo PTA (Figura 9). No processo PTA verificou-se um significativo aumento na largura do cordão, fato que se deve à utilização de um bico constritor de maior diâmetro.

A análises da Figura 9 reforçada com a análises de variância das tabelas 5, 6 e 7, indica que, o processo de soldagem e a vazão de gás de plasma apresentam efeitos significativos sobre os parâmetros geométricos do cordão. 
Tabela 1. Composição química do substrato de aço baixo carbono

\begin{tabular}{l|l|l|l|l|l|l|l|l}
\hline $\mathbf{C}$ & $\mathbf{S i}$ & $\mathbf{M n}$ & $\mathbf{P}$ & $\mathbf{S}$ & $\mathbf{C r}$ & $\mathbf{M o}$ & $\mathbf{N i}$ & $\mathbf{A l}$ \\
\hline 0,11 & 0,22 & 0,74 & 0,021 & 0,008 & 0,027 & 0,024 & 0,011 & 0,06 \\
\cline { 1 - 4 } $\mathbf{C u}$ & $\mathbf{V}$ & $\mathbf{W}$ & $\mathbf{S n}$ & $\mathbf{F e}$ & & & & \\
\cline { 1 - 4 } 0,016 & 0,015 & 0,026 & 0,065 & 98,6 & & & & \\
\cline { 1 - 3 }
\end{tabular}

Espessura : 12,7 mm

Tabela 2. Composição química do material de aporte Stellite 6 na forma de pó (BT-906)

\begin{tabular}{l|l|l|l|l|l|l|l|l}
\hline $\mathbf{C}$ & $\mathbf{S i}$ & $\mathbf{M n}$ & $\mathbf{C r}$ & $\mathbf{M o}$ & $\mathbf{N i}$ & $\mathbf{C o}$ & $\mathbf{W}$ & $\mathbf{F e}$ \\
\hline 1,32 & 1,30 & 0,028 & 30,01 & 0,24 & 2,45 & Bal & 5,21 & 2,05 \\
\hline
\end{tabular}

Dureza: 38-47 Rc; Granulometria: 45 a $150 \mu \mathrm{m}$; Densidade: 8,3 g/cm ${ }^{3}$

Tabela 3. Composição química do material de aporte Stellite 6 na forma de arame (BT-906T)

\begin{tabular}{l|l|l|l|l|l|l|l|l}
\hline $\mathbf{C}$ & $\mathbf{S i}$ & $\mathbf{M n}$ & $\mathbf{C r}$ & $\mathbf{M o}$ & $\mathbf{N i}$ & $\mathbf{C o}$ & $\mathbf{W}$ & $\mathbf{F e}$ \\
\hline $0,9-1,4$ & 2,0 & 1,0 & $26-32$ & 1,0 & 3,0 & Bal & $3,0-6,0$ & 2,0 \\
\hline
\end{tabular}

Tabela 4. Variáveis e parâmetros de soldagem

\begin{tabular}{|c|c|c|}
\hline \multicolumn{3}{|c|}{ Processo PTA } \\
\hline $\begin{array}{l}\text { Corrente de soldagem } \\
\text { Velocidade de soldagem } \\
\text { Vazão Gás de Plasma } \\
\text { Gás de proteção } \\
\text { Gás de transporte } \\
\text { Taxa de alimentação } \\
\text { Diâmetro do bico constritor/ângulo de } \\
\text { convergência } \\
\text { Distância bico-peça } \\
\text { Recuo }\end{array}$ & $\begin{array}{l}\mathrm{I}(\mathrm{A}) \\
\mathrm{Vs}(\mathrm{cm} / \mathrm{min}) \\
\mathrm{VGP}(1 / \mathrm{min}) \\
\mathrm{GPr}(1 / \mathrm{min}) \\
\mathrm{GTr}(1 / \mathrm{min}) \\
\operatorname{Tx}(\mathrm{kg} / \mathrm{h}) \\
\mathrm{d}(\mathrm{mm}) /\left(^{\circ}\right) \\
\operatorname{DBP}(\mathrm{mm}) \\
\operatorname{Rc}(\mathrm{mm})\end{array}$ & $\begin{array}{l}160 \\
20 \\
2,2 ; 2,4 ; 3,0 \\
10 \\
2 \\
1,4 \\
4,8 / 30 \\
10 \\
2,4\end{array}$ \\
\hline \multicolumn{3}{|c|}{ Processo PAW } \\
\hline $\begin{array}{l}\text { Diâmetro do arame (tubular) } \\
\text { Velocidade de arame } \\
\text { Taxa de deposição } \\
\text { Diâmetro do bico constritor }\end{array}$ & $\begin{array}{l}\mathrm{mm} \\
\mathrm{Va}(\mathrm{m} / \mathrm{min}) \\
\mathrm{Tx}(\mathrm{kg} / \mathrm{h}) \\
\mathrm{d}(\mathrm{mm})\end{array}$ & $\begin{array}{l}1,2 \\
3,0 \\
1,4 \\
3,2\end{array}$ \\
\hline $\begin{array}{l}\text { Corrente de soldagem } \\
\text { Velocidade de soldagem } \\
\text { Vazão Gás de Plasma } \\
\text { Gás de proteção } \\
\text { Taxa de alimentação } \\
\text { Distância bico-peça } \\
\text { Recuo }\end{array}$ & $\begin{array}{l}\mathrm{I}(\mathrm{A}) \\
\mathrm{Vs}(\mathrm{cm} / \mathrm{min}) \\
\mathrm{VGP}(1 / \mathrm{min}) \\
\mathrm{GPr}(1 / \mathrm{min}) \\
\mathrm{Tx}(\mathrm{kg} / \mathrm{h}) \\
\mathrm{DBP}(\mathrm{mm}) \\
\mathrm{Rc}(\mathrm{mm})\end{array}$ & $\begin{array}{l}160 \\
20 \\
2,2 ; 2,4 ; 3,0 \\
10 \\
1,4 \\
10 \\
2,4\end{array}$ \\
\hline
\end{tabular}




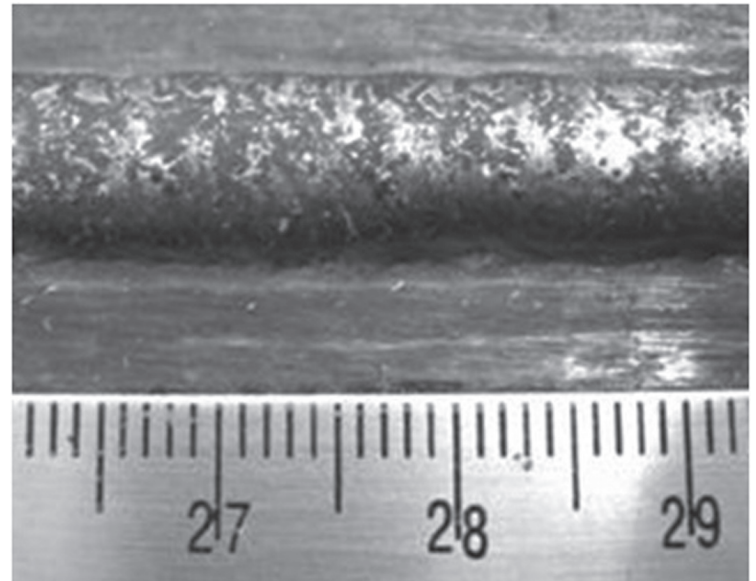

a) PAW

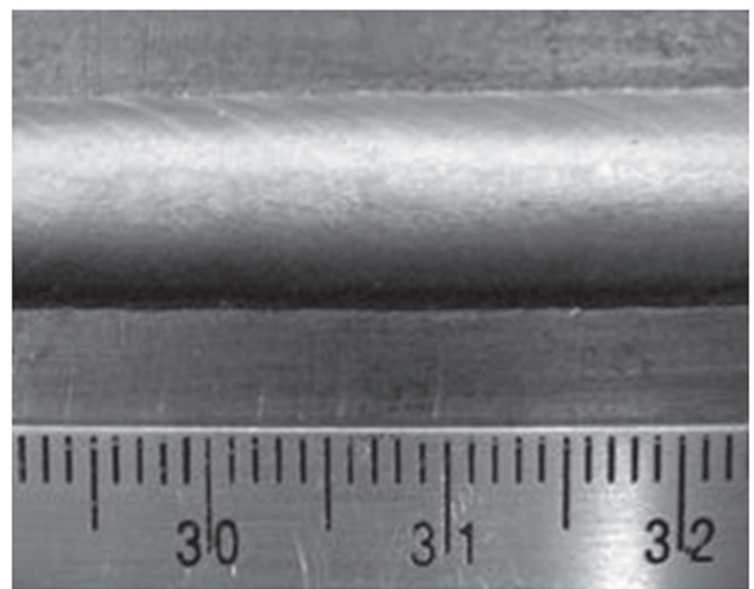

b) PTA

$\mathrm{I}=160 \mathrm{~A}, \mathrm{Vs}=20 \mathrm{~cm} / \mathrm{min}, \mathrm{Tx}=1,4 \mathrm{~kg} / \mathrm{h}$, Gás de plasma = 2,4 1/min

Figura 6. Aspecto superficial dos depósitos de Stellite 6 depositados por: a) PAW b) PTA

Com relação ao índice de convexidade ( $\mathrm{IC}=100 * \mathrm{r} / \mathrm{L})$, Silva [8] estabelece que valores próximos de $30 \%$ representam condições desejáveis para a relação largura $(\mathrm{L})$ e reforço de cordão (r). A Figura 9d apresenta o índice de convexidade do cordão de solda para o processo PAW e PTA, em função da vazão de gás de plasma.

O análises da Figura 9d, mostra que para os três níveis de vazão de gás de plasma testados, o processo PTA apresenta convexidades dos cordões aceitáveis (menores a 30\%), fato este altamente desejável. Para o caso do processo PAW, o índice de convexidade se mostra aceitável somente para níveis baixos de vazão de gás de plasma.

A análise dos valores medidos para as áreas de metal depositado indicam que há uma variação nos dois processos de soldagem estudados, o que era de esperar, em função das diferenças nos diâmetros dos bocais de constrição utilizados em cada caso e das perdas de material devido à eficiência de deposição do processo.

A Figura 10 mostra que no processo PTA se tem perdas de material. Lin [9] observou que perdas ocorrem principalmente devido à vaporização e também à dispersão das partículas depois do choque com o substrato.

Vergara [6], relata que a vazão do gás de arraste influencia na dispersão das partículas. Em muitos casos é possível, ao final da operação do revestimento, observar partículas de pó não fundidas aderidas nas laterais do revestimento. Por outro lado, em ocasiões em que a taxa de deposição é muito alta $(1,5 \mathrm{~kg} / \mathrm{h})$ em relação à corrente de soldagem (160 A) observa-se pó sem fundir espalhado sobre o substrato. Vergara [9] observou que o processo PTA apresenta uma eficiência de deposição na ordem do $87 \%$, quando é utilizado um bico constritor de $30^{\circ}$. Resultados similares são apresentados por Davis [10], mostrando uma faixa de 85 a $95 \%$ como rendimento de deposição para o processo PTA.

O gráfico da Figura 11 mostra o efeito da vazão de gás de plasma sobre o grau de diluição, utilizando-se o arame Stellite 6, diâmetro 1,2 mm tubular. Os resultados indicam que a diluição aumenta com a vazão de gás de plasma devido, talvez, à maior

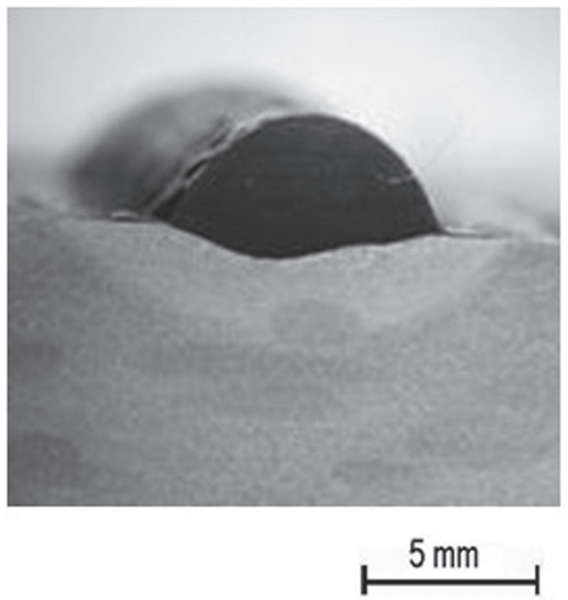

$\mathrm{VGP}=2,2(1 / \mathrm{min})$
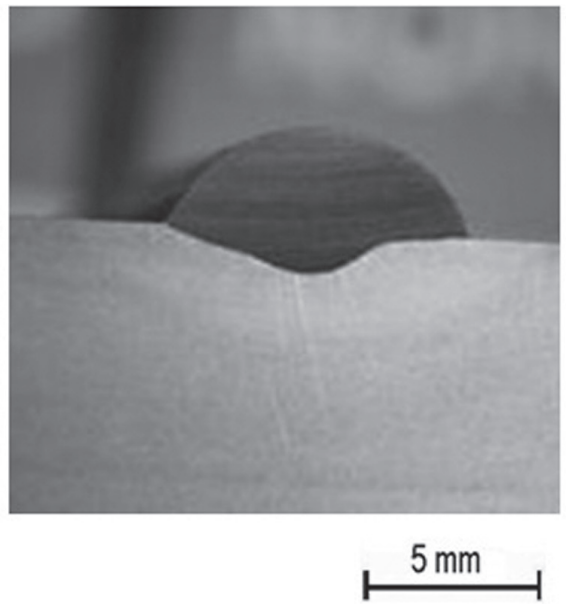

$\mathrm{VGP}=2,4(1 / \mathrm{min})$

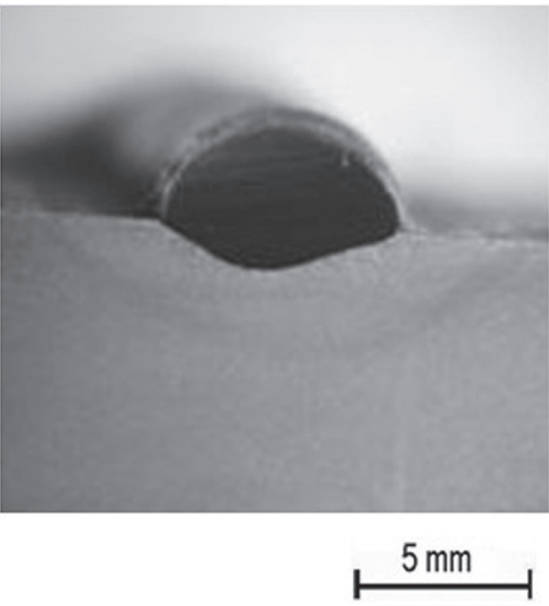

$\mathrm{VGP}=3,0(1 / \mathrm{min})$

Figura 7. Seção transversal dos cordões processados via PAW. 


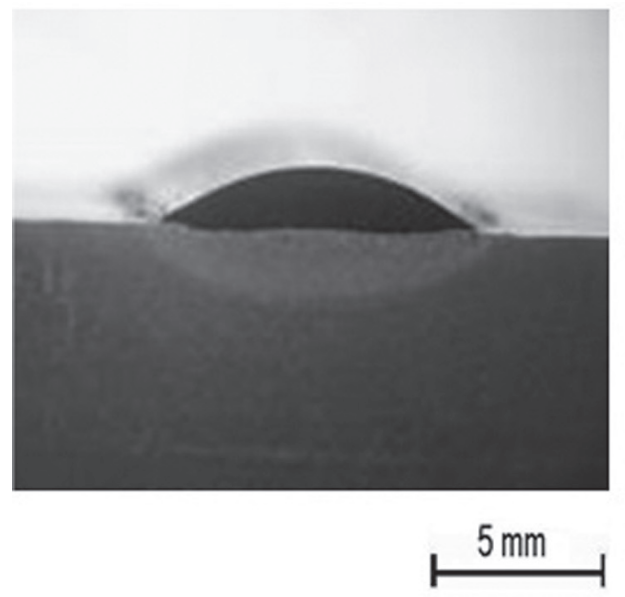

$\mathrm{VGP}=2,2(1 / \mathrm{min})$
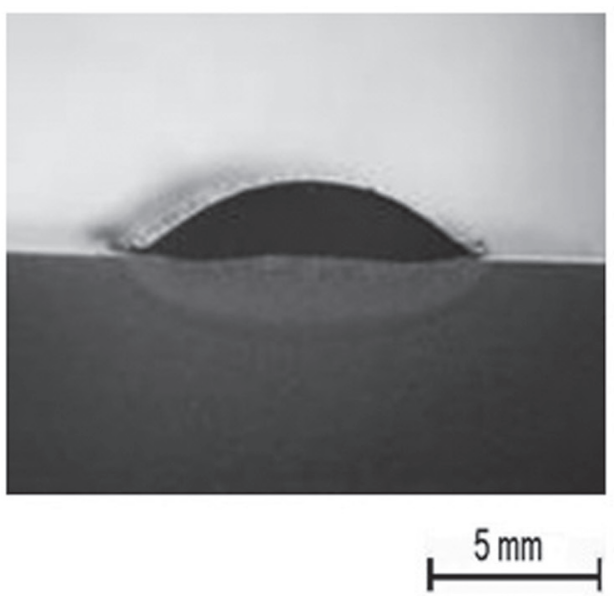

$\mathrm{VGP}=2,4(1 / \mathrm{min})$

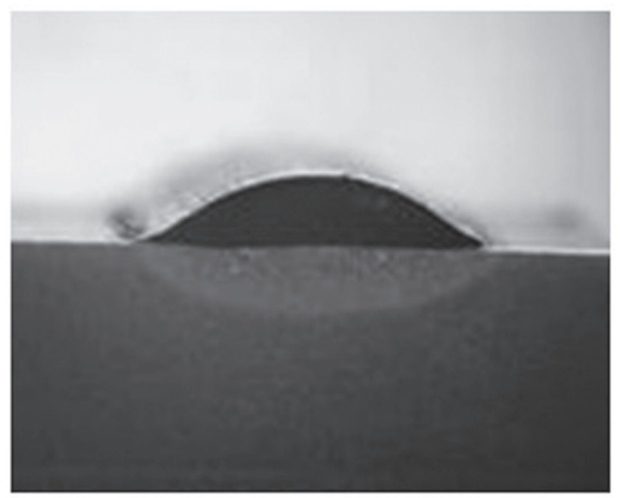

$5 \mathrm{~mm}$

$\mathrm{VGP}=3,0(1 / \mathrm{min})$

Figura 8. Seção transversal dos cordões processados via PTA.

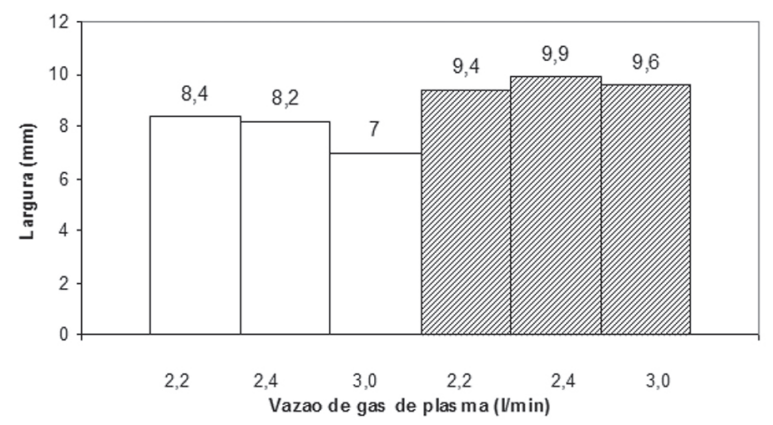

(a)

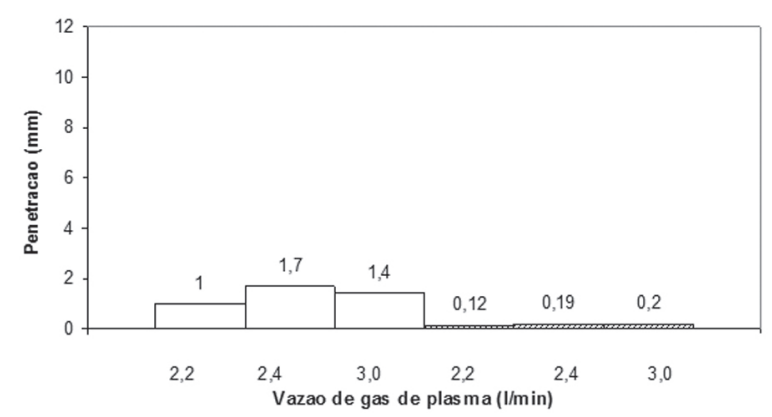

(c)

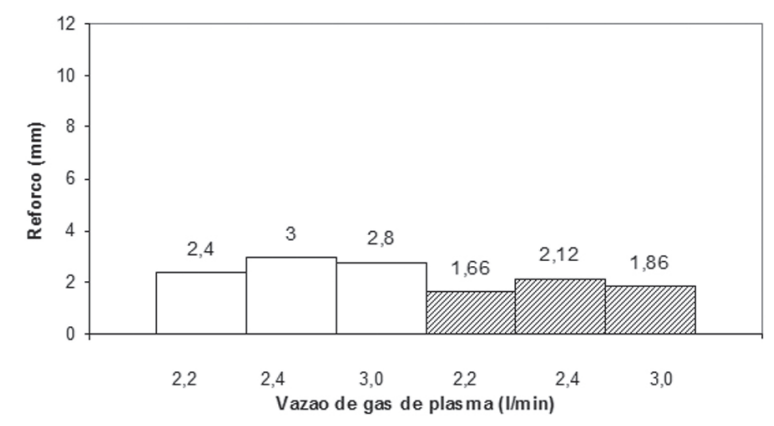

(b)

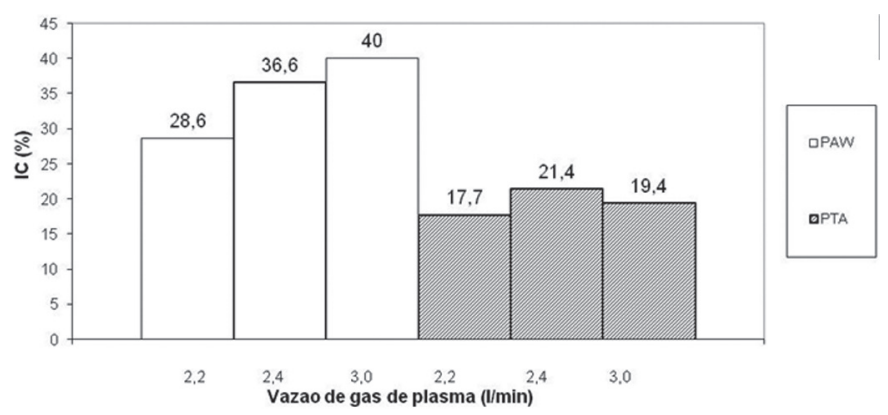

(d)

Figura 9. Efeito da vazão de gás de plasma sobre os parâmetros geométricos: a) Largura; b) Reforço; c) Penetração; d) Índice de convexidade.

pressão do jato de plasma. Resultados semelhantes foram encontrados quando se trata do processo PTA, encontrando-se valores de diluição menores aos conseguidos no processo PAW, o que era de se esperar, em função das diferenças nos diâmetros constritores dos bocais. Vergara [6] relata que o diâmetro do orifício do bico constritor tem uma grande influência sobre a qualidade do revestimento devido a que está relacionado diretamente com a largura e penetração do cordão produzido. A análise da Figura 11 reforçada com a análise de variância, Tabela 8, indica que, de uma forma geral, o processo de soldagem e a vazão de gás de plasma afeta significativamente na diluição. Conclusões similares foram relatadas por Silvério [11] envolvendo a liga Stellite 1.

Os bons resultados obtidos com o processo PTA estão associados a:

- Cordões mais largos maior área de recobrimento

- Menor diluição depósitos com composição mais próxima da liga de aporte

- Melhor molhamento, menor convexidade diminui o risco de falta de penetração/fusão entre os cordões 
Tabela 5. Resultados de análises de variância para a largura.

\begin{tabular}{lrrrrr}
\hline \multicolumn{1}{c}{ Fonte da variação } & $\begin{array}{c}\text { Soma dos } \\
\text { quadrados }\end{array}$ & $\begin{array}{c}\text { Graus de } \\
\text { liberdade }\end{array}$ & $\begin{array}{c}\text { Média dos } \\
\text { quadrados }\end{array}$ & Fobservado & F crítico \\
\hline Processo de soldagem & 17,85 & 1 & 17,85 & 1444,35 & \\
Vazão de gás de plasma & 2,316 & 2 & 1,16 & 93,67 & \\
Interação & 2,33 & 2 & 1,16 & $94,14>$ & 3,55 \\
Residual & 0,22 & 18 & 0,0124 & & \\
Total & 22,72 & 23 & & & \\
\hline
\end{tabular}

Obs.: Índice de significância $(\alpha)=5 \%$

Tabela 6. Resultados de análises de variância para o reforço

\begin{tabular}{lrrrrr}
\hline \multicolumn{1}{c}{ Fonte da variação } & $\begin{array}{c}\text { Soma dos } \\
\text { quadrados }\end{array}$ & $\begin{array}{c}\text { Graus de } \\
\text { liberdade }\end{array}$ & $\begin{array}{c}\text { Média dos } \\
\text { quadrados }\end{array}$ & Fobservado & F crítico \\
\hline Processo de soldagem & 4,29 & 1 & 4,29 & 1353,78 & \\
Vazão de gás de plasma & 1,33 & 2 & 0,66 & 209,016 & \\
Interação & 0,098 & 2 & 0,049 & $15,45>$ & 3,55 \\
Residual & 0,057 & 18 & 0,0032 & & \\
& & & & & \\
Total & 5,77 & 23 & & & \\
\hline
\end{tabular}

Obs.: Índice de significância $(\alpha)=5 \%$

Tabela 7. Resultados de análises de variância para a penetração.

\begin{tabular}{lrrrrr}
\hline \multicolumn{1}{c}{ Fonte da variação } & $\begin{array}{c}\text { Soma dos } \\
\text { quadrados }\end{array}$ & $\begin{array}{c}\text { Graus de } \\
\text { liberdade }\end{array}$ & $\begin{array}{c}\text { Média dos } \\
\text { quadrados }\end{array}$ & Fobservado & F crítico \\
\hline Processo de soldagem & 8,35 & 1 & 8,354 & 5323,15 & \\
Vazão de gás de plasma & 0,58 & 2 & 0,288 & 183,74 & \\
Interação & 0,37 & 2 & 0,185 & $118,06>$ & 3,55 \\
Residual & 0,02825 & 18 & 0,00157 & & \\
Total & 9,33 & 23 & & & \\
\hline
\end{tabular}

Obs.: Índice de significância $(\alpha)=5 \%$

Tabela 8. Resultados de análises de variância para a diluição.

\begin{tabular}{lrrrrr}
\hline \multicolumn{1}{c}{ Fonte da variação } & $\begin{array}{c}\text { Soma dos } \\
\text { quadrados }\end{array}$ & $\begin{array}{c}\text { Graus de } \\
\text { liberdade }\end{array}$ & $\begin{array}{c}\text { Média dos } \\
\text { quadrados }\end{array}$ & Fobservado & F crítico \\
\hline Processo de soldagem & 1102,43 & 1 & 1102,43 & 25289,88 & \\
Vazão de gás de plasma & 182,16 & 2 & 91,08 & 2089,39 & \\
Interação & 25,93 & 2 & 12,96 & $297,4>$ & 3,55 \\
Residual & 0,785 & 18 & 0,044 & & \\
Total & 1311,305 & 23 & & & \\
\hline
\end{tabular}

Obs.: Índice de significância $(\alpha)=5 \%$ 


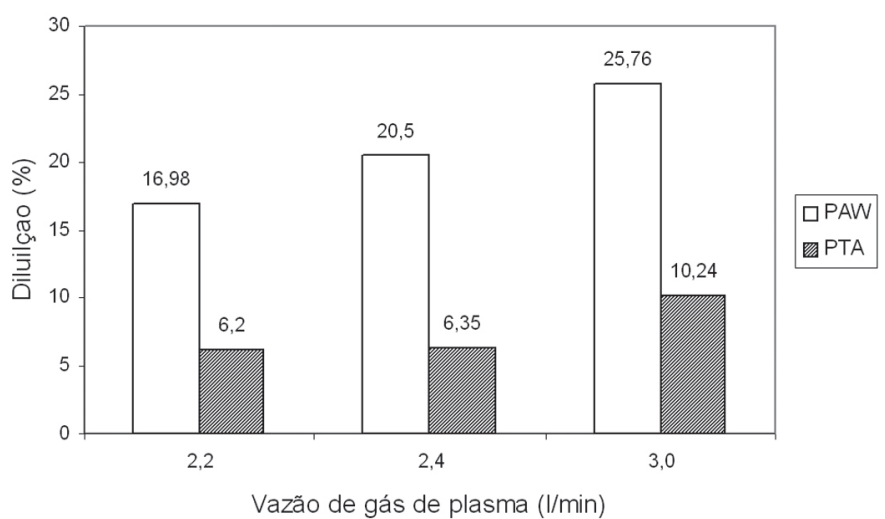

Figura 10. Área do material depositado no processo PAW e PTA.

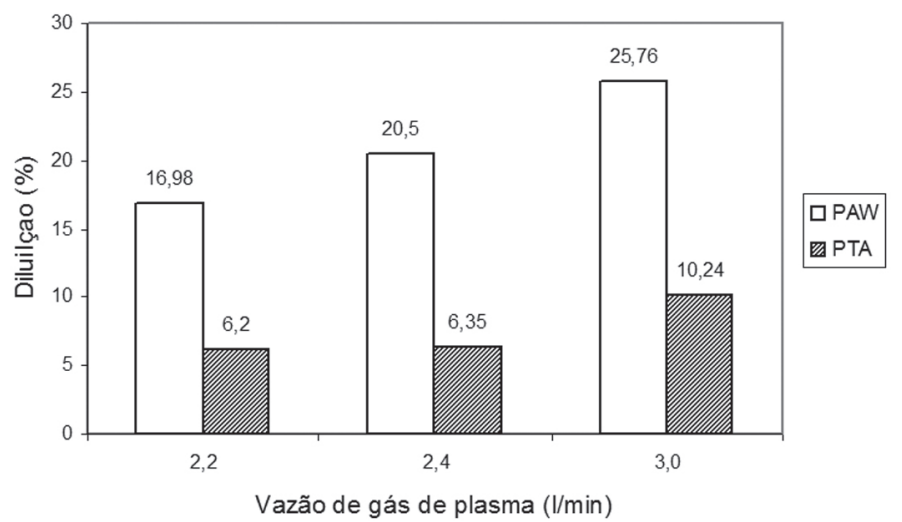

Figura 11. Efeito da vazão de gás de plasma sobre o grau de diluição no processo PAW e PTA.

Tabela 9. Resultados de análises de variância para a dureza média do MS - PAW.

\begin{tabular}{lrrrrr}
\hline \multicolumn{1}{l}{ Fonte da variação } & $\begin{array}{c}\text { Soma dos } \\
\text { quadrados }\end{array}$ & $\begin{array}{c}\text { Graus de } \\
\text { liberdade }\end{array}$ & $\begin{array}{c}\text { Médiados } \\
\text { quadrados }\end{array}$ & Fobservado & F crítico \\
\hline Vazão de gás de plasma & 18214,93 & 2 & 9107,463 & $151,9637>$ & 3,2381 \\
Residual & 2337,341 & 39 & 59,93183 & \\
& & & & \\
Total & 20552,27 & 41 & \\
\hline
\end{tabular}

Obs.: Índice de significância $(\alpha)=5 \%$

Tabela 10. Resultados de análises de variância para a dureza média do MS - PTA.

\begin{tabular}{lrrrrr}
\hline \multicolumn{1}{c}{ Fonte da variação } & $\begin{array}{c}\text { Soma dos } \\
\text { quadrados }\end{array}$ & $\begin{array}{l}\text { Graus de } \\
\text { liberdade }\end{array}$ & $\begin{array}{l}\text { Média dos } \\
\text { quadrados }\end{array}$ & Fobservado & F crítico \\
\hline Vazão de gás de plasma & 2729,185 & 2 & 1364,593 & $2,388627<$ & 3,554561 \\
Residual & 10283,17 & 18 & 571,2875 & \\
& 13012,36 & 20 & & \\
Total & 20 & & \\
\hline
\end{tabular}

Obs.: Índice de significância $(\alpha)=5 \%$

\subsection{Microdureza e microestructura}

A Figura 12 mostra as microestruturas típicas de solidificação no centro do cordão. Quando são empregados níveis de vazão de gás de plasma de $2,2 \mathrm{l} / \mathrm{min}$, em ambos os processos, a microestrutura é mais fina. Para níveis de vazão de gás de plasma de 3,0 1/min, tanto para o processo PAW e PTA, a microestrutura apresentou-se mais grosseira.

O perfil de microdureza avaliado ao longo da seção transversal dos depósitos é mostrado nas Figuras 13 e 14, para o Processo PAW e o Processo PTA, respectivamente.

A análise da Figura 13 reforçada com a análise de variância, Tabela 9, indica que, de uma forma geral, a vazão de gás de plasma afeta significativamente na dureza. Por outro lado, a análise da Figura 14, que corresponde ao processo PTA, reforçado com a Tabela 10, indica que a vazão de gás de plasma não afeta significativamente na dureza. Depósitos processados com o processo PAW, produziram valores de dureza menores, conforme esperado pelas estruturas mais grosseiras e maiores graus de diluição.

Comprovou-se que o processo PTA gera uma microestrutura mais refinada e conseqüentemente durezas mais elevadas que o processo PAW, fato que também foi observado por Silvério [11]. 


\section{PAW}

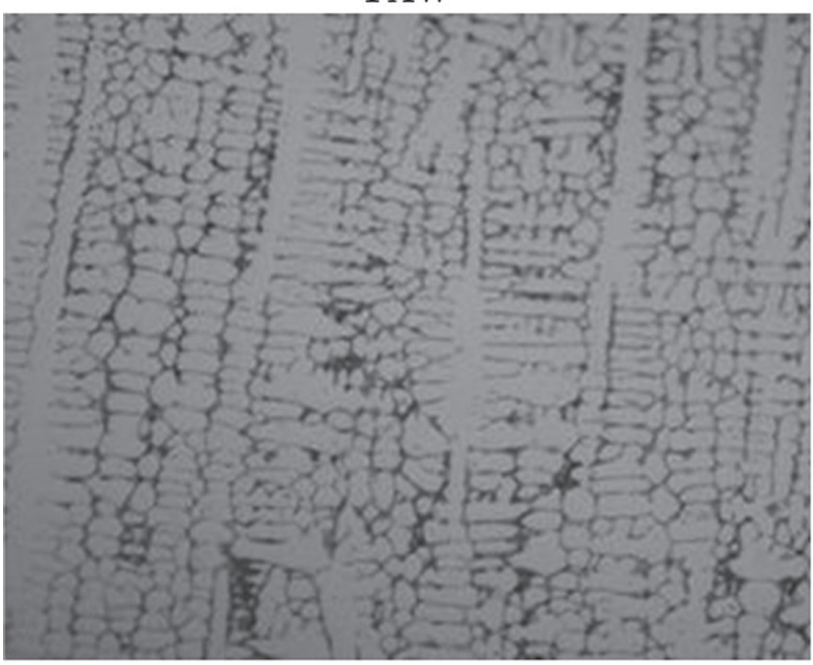

PTA

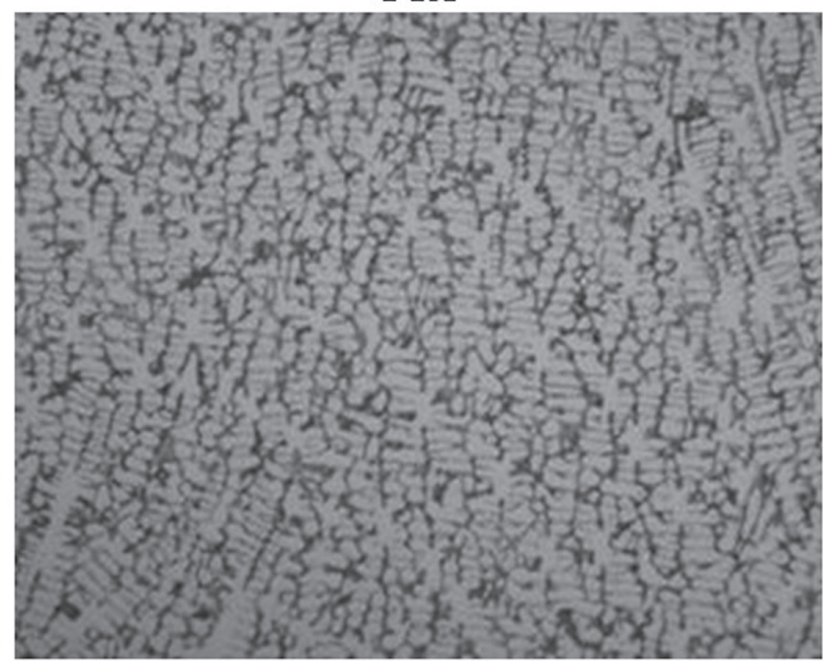

a) Vazão de Gás de Plasma (VGP) = 3,0 (1/min)

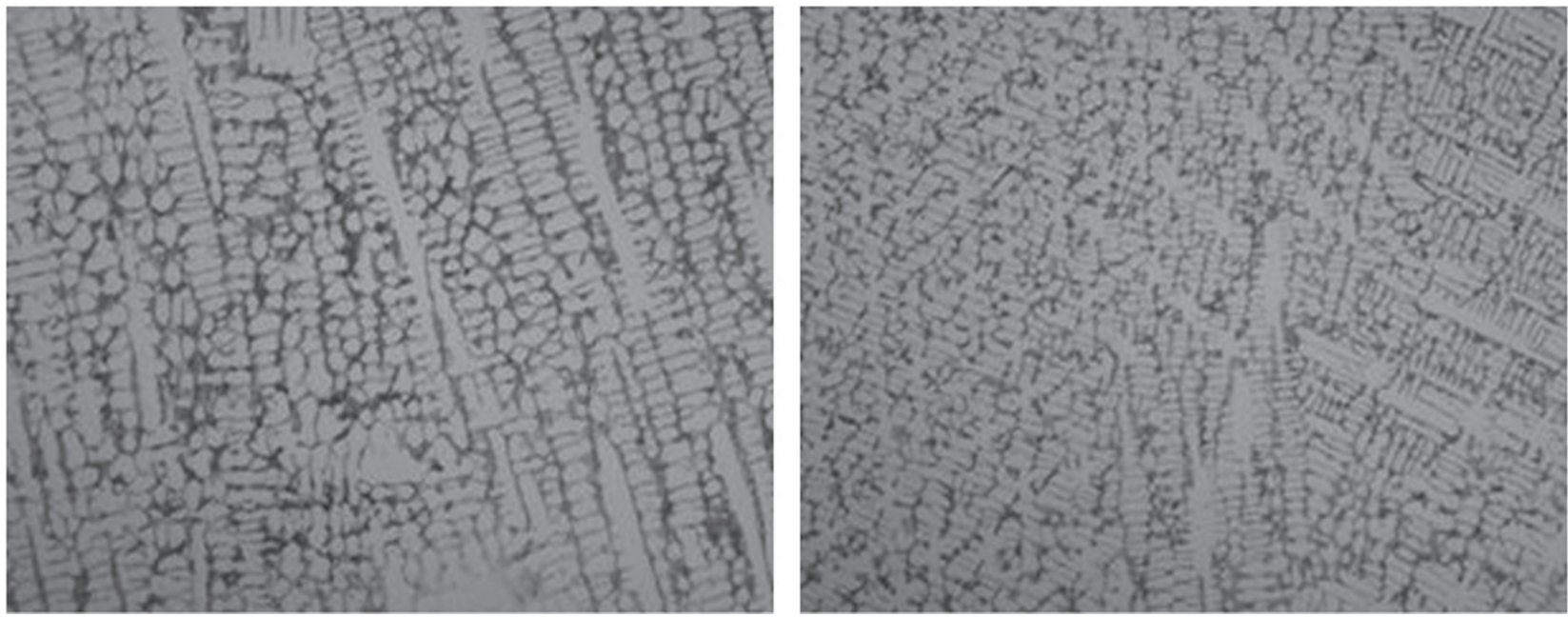

b) Vazão de Gás de Plasma (VGP) = 2,4 (1/min)
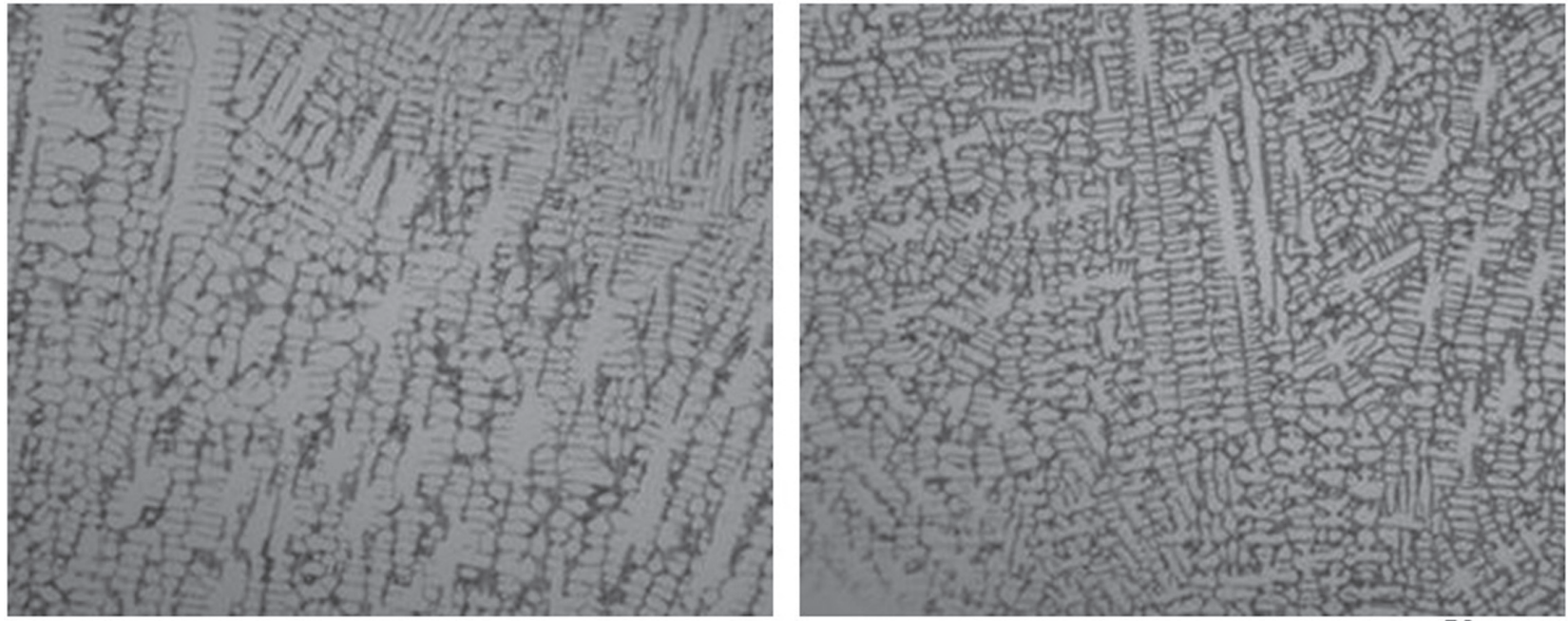

$50 \mu \mathrm{m}$

c) Vazão de Gás de Plasma ( VGP) = 2,2 (1/min)

Figura 12. Micrografias das amostras de Stellite 6 para o Processo PAW e PTA. Centro do cordão. 
Pocesso-PAW

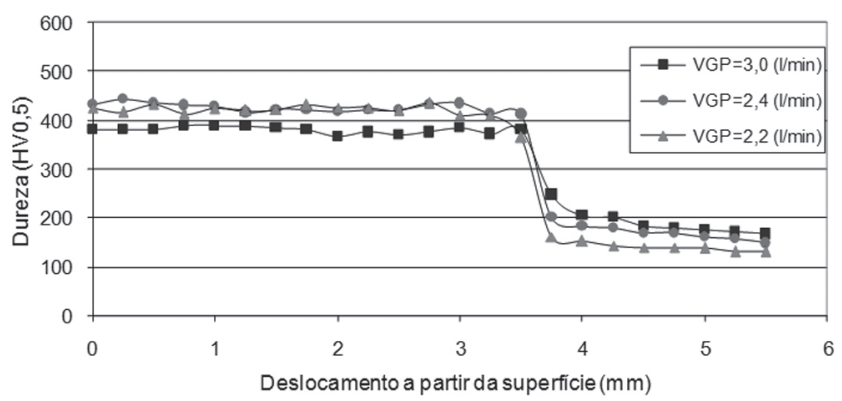

Figura 13. Efeito da vazão de gás de plasma sobre a dureza no processo PAW.

Processo-PTA

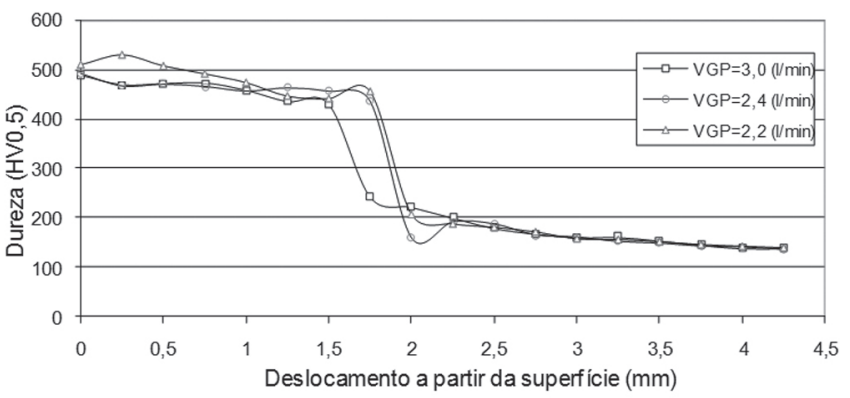

Figura 14. Efeito da vazão de gás de plasma sobre a dureza no processo PTA.

\section{Conclusões}

Com base nos resultados experimentais obtidos neste trabalho as conclusões são as seguintes:

- O processo PTA foi o que produziu o melhor acabamento superficial e melhor molhamento.

Em função da eficiência de deposição e da diferença no diâmetro do orifício do bico constritor utilizado, nos processos de soldagem estudados, os principais resultados são:

- No processo PTA foram encontrados valores de diluição menores aos conseguidos no processo PAW.

- Maior largura foi conseguida pelo processo PTA.

- Quando comparados os depósitos realizados através dos dois processos, pode-se observar que o reforço e penetração são sempre menores no processo PTA.

- Depósitos processados com o processo PAW, produziram valores de dureza menores, conforme esperado pelas estruturas mais grosseiras e maiores graus de diluição.

\section{Referências bibliográficas}

[1] DAI, W. S.; CHEN, L. H.; LUI, T. S. $\mathrm{SiO}_{2}$ particle erosion of spheroidal graphite cast iron after suface remelting by the plasma transferred arc process. Disponível em:<http://www. sciencedirect.com> Acesso em: Nov. 2008.
[2] GATTO, A.; BASSOLI, E.; FORNARI, M. Plasma Transferred Arc deposition of powdered high performances alloys: process parameters optimisation as a function of alloy and geometrical configuration. Disponível em:<http://www. sciencedirect.com> Acesso em: Jun. 2009.

[3] ZHANG, L. et al. Effect of niobium on the microstructure and wear resistance of iron-based alloy coating produced by plasma cladding. Disponível em:<http://www.elsevier.com/ locate/msea> Acesso em: Nov. 2008.

[4] LIU, Y. F. et al. Microstructure and dry-sliding wear properties of TiC-reinforced composite coating prepared by plasmatransferred arc weld-surfacing process. Disponível em: $<$ http:// www.elsevier.com/locate/msea> Acesso em: Nov. 2008.

[5] M. A. Oliveira. Estudo do processo plasma com alimentação automática de arame: 2001. 78p. Dissertação (Mestrado em Engenharia Mecânica)-Programa de Pós-Graduação em Engenharia Mecânica, UFSC, Florianópolis.

[6] VERGARA, V. M. Inovação do equipamento e avaliação do processo plasma de arco transferido alimentado com pó (PTAP) para soldagem fora de posição: 2005. 174p.Tese (Doutorado em Engenharia Mecânica) - Programa de Pós-Graduação em Engenharia Mecânica, UFSC, Florianópolis.

[7] HALLEN, H.; LUGSCHEIDER, E.; AIT-MEKIDECHE, A. Plasma transferred arc surfacing with high deposition rates. In: Proceedings of conference on thermal spray coatings: properties. processes and applications, Pittsburgh, USA, 4-10 May 1991. ASM International; 1992. p. 537-9.

[8] SILVA, César Rezende; FERRARESI, Valtair Antonio; SCOTTI, Américo. A quality and cost approach for welding process selection. J. Braz. Soc. Mech. Sci., Campinas, v. 22, n. 3, 2000 . Available from <http:// www.scielo.br/scielo.php? script=sci_arttext\&pid=S0100$73862000000300002 \& \operatorname{lng}=\mathrm{en} \& \mathrm{nrm}=\mathrm{iso}>$. access on 29 Nov. 2009. doi: 10.1590/S0100-73862000000300002. Acesso em: Nov. 2009.

[9] LIN, J. A. Simple model of powder catchment in coaxial laser cladding. Optics \& Laser Technology, 1999, 233-238

[10] DAVIS, J. R. - Davis and Associates. Hardfacing, Weld Cladding and Dissimilar Metal Joining. In: ASM Handbook - Welding, Brazing and Soldering, Vol. 6. 10 $0^{\text {th }}$ ed. OH: ASM Metals Park, 1993. p. 699-828.

[11] R. B. Silvério, A.S. C. M. D'Oliveira. Revestimento de Liga a Base de Cobalto por PTA com Alimentação de Pó e Arame. In: Congresso Brasileiro de Engenharía de Fabricação, UberlândiaMG, Maio. 2003. 